\title{
Goals in Social Information Processing: The Case of Anticipated Interaction
}

\author{
Patricia G. Devine and Constantine Sedikides \\ University of Wisconsin-Madison \\ Robert W. Fuhrman \\ Ohio State University
}

\begin{abstract}
Examined the role of anticipated-interaction instructions on memory for and organization of social information. In Study 1, Ss read and recalled information about a prospective partner (i.e., target) on a problem-solving task and about 4 other stimulus people. The results indicated that (a) Ss recalled more items about the target than the others, (b) the target was individuated from the others in memory, and (c) Ss were more accurate on a name-item matching task for the target than for the others. Study 2 compared anticipated interaction with several other processing goals (i.e., memory, impression formation, self-comparison, friend-comparison). Only anticipated-interaction and impression formation instructions led to higher levels of recall and more accurate matching performance for the target than for the others. However, the conditional probability data suggest that anticipated interaction led to higher levels of organization of target information than did any of the other conditions. Discussion considers information processing strategies that are possibly instigated by anticipated-interaction instructions.
\end{abstract}

Imagine that you are attending your first day of classes, and students are nervously and excitedly introducing themselves to each other. On this occasion, you spot your "dream date." Although there are several people around, the prospective date may take on particular importance to you because you hope to have future interactions with him or her. The importance assigned to the dream date may affect what you remember about him or her and about the other students in the class and how that information is organized in memory.

Alternatively, imagine that you are interviewing a job candidate. The very real possibility of future interaction with this person may influence how you think about this person, what you remember about him or her, and how you organize that information.

These examples suggest that the goals individuals bring to bear on social situations may have an important effect on their perceptions of and memory for others. The present research, with its focus on how goals affect social information processing, reflects the renewed interest in the interaction between motivation and cognition in social perception (Neuberg \& Fiske, 1987; Sorrentino \& Higgins, 1986; Srull \& Wyer, 1986). The extant research on goals in person memory, however, focuses primarily

\footnotetext{
We thank John Skowronski, Eliot Smith, and several anonymous reviewers for very helpful comments on earlier drafts of this article. We also thank Deborah Weaver for her help in data collection and coding for Study 1 and Roderick Young for his assistance in Study 2. Robert Fuhrman is now at the University of Lllinois at Urbana-Champaign.

Study 1 was presented at the 95th Annual Convention of the American Psychological Association, New York, August 1987.

Correspondence concerning this article should be addressed to Patricia G. Devine, Department of Psychology, University of Wisconsin, 1202 West Johnson Street, Madison, Wisconsin 53706
}

on the effect of impression formation versus memory instructions on subjects' encoding, storage, and retrieval processes. It is well established, for example, that impression formation instructions lead to better recall and more effective organization of stimulus information than do memory instructions (Hamilton, Katz, \& Leirer, 1980; Pryor, Simpson, Mitchell, Ostrom, \& Lydon, 1982; Srull, 1981; Wyer \& Gordon, 1982). Although this research convincingly demonstrates that perceivers' goals for processing social information substantially affect their cognitive processing, the generality of research examining the relation between perceivers' goals and cognitive processing is limited.

Until recently, there has been little theorizing or empirical research on the effects of expecting future interactions with stimulus people on subjects' cognitive processing of and memory for information about these people (Hastie, Park, \& Weber, 1984). Much of the previous anticipated-interaction research focused primarily on the effects of anticipated interaction on liking (Berscheid, Boye, \& Darley, 1968; Berscheid, Graziano, Monson, \& Dermer 1976; Layton \& Insko, 1974; Tyler \& Sears, 1977), impression formation (Erber \& Fiske, 1984; Neuberg \& Fiske, 1987), or attribution (Harvey, Yarkin, Lightner, \& Town, 1980). For example, Tyler and Sears (1977) found that anticipated-interaction instructions increased subjects' liking for targets who were initially dislikable or ambiguous, but did not affect their liking for initially likable targets. None of these studies on impressions or attributions were designed specifically to explore the hypotheses addressed in the present research. That is, although some of the studies assessed memory (e.g., Berscheid et al., 1976; Harvey et al., 1980), their focus was not on the organization of memory or the nature of memory representations.

In contrast to these studies, recent research by Srull and 
Brand (1983) has begun to explore the effects of anticipatedinteraction instructions specifically on person memory. Srull and Brand's focus was on possible encoding differences that might result from various learning strategies or processing objectives. Srull and Brand (1983) compared anticipated-interaction instructions (i.e., you will meet one of the stimulus people) with memory instructions (i.e., remember the items for a recall test) on how much subjects recalled about stimulus people and how that information was organized in memory. They argued that when subjects believe that they will actually meet one of the people to work on some task, they will try to integrate the information learned about each person into a coherent impression of that person and attempt to keep the information pertaining to each person separate from that learned about the other people. Srull and Brand found, as they had predicted, that overall levels of recall were higher following anticipated-interaction instructions than following memory instructions. In addition, subjects appeared to be more likely to organize information around the individuals in memory if future interaction was anticipated than if it was not.

Srull and Brand's (1983) findings represent a useful initial step into exploring the effects of anticipated-interaction instructions on subjects' processing of and memory for information about others. Their findings suggest that anticipating interaction with others is a powerful manipulation that is likely to lead subjects to individuate or personalize stimulus people. However, Srull and Brand's findings need to be qualified.

First, Srull and Brand (1983) confounded anticipated-interaction with impression formation instructions. That is, subjects in the anticipated-interaction conditions were specifically instructed "that they should form an impression of what the individual would be like because they would later meet him (or one of them)" (p. 203). As a result of this confounding, it is difficult to separate the effect of anticipating interaction with a target from that of simply forming an impression of the target. Second, Srull and Brand's study involved only a two-person stimulus field. Although not necessarily a limitation, in many situations one is confronted with much more complex stimulus fields (e.g., meeting several unfamiliar people) in which one or more people may be identified as possible targets of future interaction. Finally, Srull and Brand provided no evidence independent of the recall data concerning the effectiveness of the anticipated-interaction instructions. It should be noted, however, that their research was not designed to address the concerns of the present research. Their primary goal was to examine whether the parameters of the Search of Associative Memory model are sufficient to model social psychological phenomena.

Our interest in the present research was in developing an understanding of the effects of anticipated-interaction instructions on memory for and organization of information learned about other people. Whereas Study 1 examined only the effect of anticipated-interaction instructions, Study 2 compared anticipated-interaction instructions with a variety of other instructions in an effort to determine what subjects do when they anticipate interacting with one of several target people. In both studies reported in this article, we manipulated anticipated interaction independently of impression formation (i.e., subjects were not instructed to form an impression as part of the anticipated-interaction instructions). However, only in Study 2 did we compare anticipated-interaction instructions with impression formation instructions. Moreover, we made an effort to enhance the experimental realism of our research. Subjects were led to believe that the information they would learn was generated by and descriptive of the other subjects participating in the study. As a result, subjects believed they were learning about actual people, one of whom they would subsequently interact with on a group problem-solving task. Unlike Srull and Brand's (1983) research, which addressed the issue of whether subjects can individuate targets, the present research explored whether subjects can selectively individuate a target within a group.

\section{Study 1}

In Study 1, all subjects were led to expect that they would interact with one of five other people on a problem-solving task. They were told that they would have an opportunity to learn information about their prospective partner and about the others that would be useful to them during the problem-solving session. Similarly to Srull and Brand (1983), we expected that if subjects believe that they will actually meet and interact with one of the people, it would be functional for them to cognitively individuate the partner from the other stimulus people. Because the subjects will have to work with only the partner, it is crucial that they get a clear sense of what type of person the partner is and what type of characteristics the partner possesses that might lead to a productive group problem-solving session and a pleasant interaction. The stimulus people with whom there is no expected interaction would be of less immediate concern for subjects. Subjects would not be dependent on these others in the upcoming interaction, and therefore it may not be as important for them to cognitively individuate these stimulus people from each other (Erber \& Fiske, 1984; Neuberg \& Fiske, 1987).

The notion of "immediate concern" is ambiguous; we would like to go beyond it and speculate briefly on possible socialcognitive mechanisms through which anticipated-interaction instructions could influence memory. Anticipated-interaction instructions may lead subjects to devote greater levels of online attention to the partner than to the other stimulus people. Alternatively, anticipated-interaction instructions may cause subjects to elaborate more fully on the information about the partner (e.g., making inferences, comparing partner stimulus items with one another) either at the initial encoding phase or at the later phases of information processing.

The differences in the way information about the partner versus about the other stimulus people is processed should lead to three outcomes. First, the expectation of future interaction should encourage subjects to form more name-to-item associations for the partner than for the other stimulus people. This suggests that subjects would be better at matching a name with an item in a subsequent matching task when the items pertain to the partner than when they pertain to the other stimulus people (cf. Pryor \& Ostrom, 1981). Second, the anticipation of an interaction should encourage subjects to think more precisely about how the pieces of information about the partner are related to each other. This should lead subjects to form more item-to-item associations for information about the partner than about the others. As a result, in a free-recall task, information about the target will tend to be recalled contiguously much 
Table 1

Stimulus Sets Used in the Studies

\begin{tabular}{|c|c|c|c|c|c|}
\hline \multirow[b]{2}{*}{$\begin{array}{l}\text { Stimulus } \\
\text { person }\end{array}$} & \multicolumn{5}{|c|}{ Stimulus Set 1} \\
\hline & Major & Traits & Pet peeve & Hometown & $\begin{array}{l}\text { Favorite } \\
\text { performer }\end{array}$ \\
\hline Mary & $\begin{array}{l}\text { Computer } \\
\text { information } \\
\text { sciences }\end{array}$ & Honest & Smoking & Columbus & Lionel Richie \\
\hline Tina & Psychology & Friendly & Interrupting & Loraine & Duran Duran \\
\hline Susan & $\begin{array}{l}\text { Business } \\
\text { administration }\end{array}$ & Emotional & Knuckle crack & Toledo & Madonna \\
\hline Barb & Music education & Jealous & Chew loudly & Akron & Diana Ross \\
\hline \multirow[t]{3}{*}{ Ann } & English & Loudmouth & Gossiping & Cincinnati & Bryan Adams \\
\hline & \multicolumn{5}{|c|}{ Stimulus set 2} \\
\hline & $\begin{array}{l}\text { Favorite } \\
\text { game }\end{array}$ & Trait-Others & Hobby & $\begin{array}{l}\text { Place } \\
\text { of living }\end{array}$ & $\begin{array}{l}\text { Part-time } \\
\text { job }\end{array}$ \\
\hline Carol & Solitaire & Independent & Reading & Chittenden & Waitress \\
\hline Patty & Risk & Aggressive & Piano & Arlington & Lifeguard \\
\hline Sandy & Clue & Argumentative & Running & Norwich & Cashier \\
\hline Lisa & Monopoly & Unemotional & TV & Morril Tower & Secretary \\
\hline Debora & Scrabble & Sociable & Gourmet cook & 17th Avenue & $\begin{array}{l}\text { Gas station } \\
\text { attendant }\end{array}$ \\
\hline
\end{tabular}

more so than would information about each of the remaining stimulus people. This hypothesis can be explored by examining the conditional probabilities associated with recalling items from the partner category with the average conditional probability associated with recalling items from the four nonpartner stimulus person categories. The conditional probability index should be higher for the partner as compared with the average conditional probability index for the remaining stimulus people. Finally, we expect that the greater amount of organization of information learned about the partner in the form of the name-to-item and item-to-item associations will produce a higher level of recall for the partner as compared with that for the other stimulus people.

\section{Method}

Subjects and design. Subjects were 24 female introductory psychology students who participated in the study for course credit. All subjects expected to interact with one of the stimulus people on a problem-solving task. However, the study included a number of between-subjects variables for purposes of counterbalancing and generalizability. Thus, the study's design was a Stimulus Set (one vs. two) $\times$ Partner (A vs. B) $\times$ Partner Location in the Stimulus Booklet (second vs. fourth) factorial. We randomly assigned 3 subjects to each of the eight conditions.

Stimulus materials. We constructed two stimulus sets (see Table 1). Each set contained five information items about each of five stimulus people. The information items were from five different categories. Subjects were told that this information might be useful to them in the problem-solving session. The five categories corresponded to questions that the subjects had previously responded to in a telephone interview (see the following paragraph). In Set 1 the subjects learned about each stimulus person's major, self-descriptive trait, pet peeve, hometown, and favorite musical performer. In Set 2 subjects learned about each stimulus person's favorite board game, hobby, place of residence, and parttime summer job and a trait others use to describe the person. The state- ments were organized into a booklet with one statement on each page. Each information item was associated with a stimulus person's name (e.g., Barb's major is music education). We organized the order of information items within the stimulus set on a person by person basis; however, the order of the categories was random within each person.

Procedure. Subjects read about a person perception study on a signup board and called the experimenter for an appointment. When the subject called, she was told that the study involved subjects participating in small groups and that in an effort to coordinate the groups the experimenter would like to ask her to respond to a few general information questions (e.g., major, pet peeve, favorite board game, etc.). All subjects complied with this request. After responding to the questions, the subject scheduled an appointment for the study. The purpose of the telephone interview was to enhance the believability of the experimental session, during which subjects would learn about ostensibly real others. In actuality, subjects' responses to the questions were not recorded and the people about whom subjects learned were fictitious.

Subjects were run in groups ranging in size from 3 to 6 people. On arrival, subjects were escorted to the experimental room one by one so that they could not interact with one another in the waiting area. The experimental room was set up with a partitioned table so that subjects could not tell how many people were in the room. They were led to believe that when the experiment began there were five other people in the experimental room.

After subjects were seated at the partitioned table, the experimenter asked them to read through a set of instructions. These instructions indicated that the major goal of the research was to study the ways in which groups composed of two people who have had no prior contact with each other work together to solve problems. Subjects were led to believe that they would be assigned to a group with one of the other participants. They were then told that the study would occur in two sessions. The second session was to be the two-person problem-solving session. Each group would work together for 25-30 min to solve several different kinds of problems. The problems would require cooperation between the two group members (the specific nature of the problems was to be revealed later). The subjects were told that their performance 
would be compared with and evaluated against each of the other twoperson groups.

The instructions went on to suggest that previous research had shown that success on the group problem-solving task was dependent on (a) being familiar with group members and (b) cooperative efforts. Thus, in the first session of the study subjects would be given an opportunity to learn information about their prospective partners that would be useful to them during the problem-solving task. They were also told that they would be provided with information about the other people present so that they would have an idea of what those people were like as compared with their partner.

Subjects were told that the information they would learn about their partner and the other people was based on the telephone interviews they had participated in when they called to schedule their appointment for the study. The experimenter explained that their responses to the general information questions had been compiled into booklets. Each subject was told that her booklet contained information about the other subjects participating in the study. Subjects were assured that only their first names were used in the booklet and that their last names would remain confidential. Before beginning the experiment, subjects were given an opportunity to object to having their information put into a booklet, but none of them did.

Subjects were then paced through the 25-page booklet containing the stimulus items at a rate of $6 \mathrm{~s}$ per page to control for on-line attention (Neuberg \& Fiske, 1987). Their partner was identified as being either the second or the fourth person described in the stimulus booklet. A tape recording signalled the subjects to turn the pages of the booklet. They were instructed to read through each stimulus item once and not to turn back to any previous page in the booklet. After reading the stimulus items, subjects were told that one of the group problem-solving tasks involved geography. In preparation for the group task, they were asked to generate some information individually. Their task involved listing as many of the United States as they could remember. This task served as a distractor task to eliminate any short-term memory effects on the free recall of the stimulus items. Subjects were given approximately $2.5 \mathrm{~min}$ for this task.

Subjects then completed the primary dependent measures for the study. They were provided with a blank booklet and asked to write down as many descriptions about all five stimulus people as they could remember. They were asked to write only one item per page, to write the items in any order, and to indicate the people's names whenever possible. Once they had turned a page they were asked not to turn back to any previous page. Subjects were told that it was important for the experimenters to know how much information the subjects remembered about each of the people prior to the problem-solving task. Subjects were given 5 min for the recall task, which was ample time for all subjects to complete the task. Following the memory task, subjects completed a name-to-stimulus item matching task. Subjects were given a list of the stimulus people's names and a list of the items that they had previously read. They were asked simply to fill in the name that had previously been associated with each item.

In the period following the matching task, subjects completed a number of manipulation check items. First, subjects were asked to list all the thoughts that were going through their minds concerning their next task, which involved solving problems with their partner. They were asked to write one thought per page in a booklet without turning back to any previous page. In addition, subjects were asked to indicate whether they understood their objective in reading through the stimulus materials and whether they had followed that objective. They were asked to indicate whether they indeed anticipated interacting with others on problem-solving tasks and to indicate the number of people with whom they expected to interact.

Finally, subjects were fully debriefed. The goals of the study were described, and subjects were informed that the experimenter had never recorded their responses to the telephone interviews and that the people they had read about in the stimulus materials were actually fictitious. Subjects were told that the purpose of using the telephone interviews was to enhance the realism of the task.

\section{Results}

Effectiveness of the anticipated-interaction instructions. Examination of the manipulation check questions indicated that the anticipated-interaction instructions were successful. In response to the question asking subjects whether they had believed that they were going to participate in a problem-solving session, $92 \%$ of the subjects said yes, only $8 \%$ of the subjects said no, and all subjects accurately reported the number of persons with whom they expected to interact. ${ }^{1}$ In addition, $96 \%$ of the subjects reported that they had understood and followed their instructions.

Finally, examination of the thoughts that were going through subjects' minds prior to the group problem-solving session suggested that subjects were thinking about the upcoming problem-solving session, about their prospective partner, and about the likelihood that the interaction would go smoothly. Interestingly, 54\% of the subjects expressed somewhat negative feelings about the ensuing interaction. That is, they were nervous and wondered if the partner would like them or whether they would do well on the problem-solving task. In contrast, the remaining $46 \%$ of the subjects expressed positive feelings about the upcoming interaction. They expected that the group problem-solving session would be fun and looked forward to meeting and working with their partner. Whether their immediate thoughts were positive or negative, their thoughts were certainly focused on the anticipated interaction with their prospective partner and the problem-solving task.

Memory and organization measures. ${ }^{2}$ We scored free-recall protocols according to a gist or general meaning criterion. From subjects' recall protocols, we calculated the total number of items that subjects correctly recalled and also the conditional probability scores. In addition, we examined subjects' performance on a name-to-item matching task.

From subjects' recall protocols, we calculated the number of items that subjects recalled about each stimulus person and compared recall for the partner with the average number of items recalled for the other four stimulus people. Consistent

\footnotetext{
'All of the subsequent analyses reported in this article were done both including the subjects who did not believe that they would interact with another person during the experiment and eliminating these subjects. Eliminating these subjects did not change the pattern of the results. The analyses reported in the text include these subjects.

${ }^{2}$ In addition to the primary analyses, we examined each of the memory and organization scores as a function of subjects' feelings reported as they waited for the group problem-solving task to begin. That is, Positive versus Negative Feelings was introduced as a factor in the analyses of the recall, conditional probability, and matching scores for the partner as compared with the average scores for the remaining four stimulus people. Whether subjects were feeling negatively or positively did not alter the overall pattern of results observed for subjects' recall, conditional probability, or matching scores. Feeling type did enter into a couple of higher-order interactions, but these interactions are uninterpretable.
} 
with expectations, recall was greater for the partner $(M=3.71)$ than for the others $(M=2.28), F(1,16)=30.62, p<.00001$.

In addition to our interest in subjects' ability to recall stimulus items, we were also concerned with the organization of that information in memory. We expected that subjects would be more likely to organize relevant information about the partner than about the other stimulus people. To explore this hypothesis, we calculated a conditional probability index for each stimulus person from subjects' recall protocols. A conditional probability index provides a measure of the extent to which after recalling an item about a person the next item recalled is also about that person. A conditional probability index controls for number of items recalled. As expected, the conditional probability index for the partner (i.e., the probability of recalling an item about the partner given that another item about the partner has just been recalled) was greater $(M=.32)$ than the average conditional probability index for the other four stimulus people $(M=.09), F(1,16)=7.01, p<.02$.

Finally, we expected that subjects' performance on the nameto-item matching task would be better for the partner than for the other stimulus people. This prediction was supported. Subjects were more accurate in matching names with items for the partner $(M=2.88)$ than for the other stimulus people $(M=$ $1.88), F(1,16)=8.80, p<.009$. Each of the recall, matching, and conditional probability findings was robust, generalizing across the stimulus set, partner, and partner location conditions.

In the analyses just summarized, we compared recall, conditional probability, and matching data for the partner with the average of the scores for the remaining four stimulus people. It is possible that these averages biased the findings in favor of our hypotheses (i.e., the previous comparison could have been distorted by including the least well remembered and the least well organized of the stimulus persons). To correct for this possibility, we did a series of analyses in which we compared the recall, conditional probability, and matching scores of the partner with the scores of the best remembered and best organized of the remaining four stimulus people. Recall for the partner $(M=3.71)$ was better than for the best recalled of the other stimulus people $(M=2.46), t(24)=4.05, p<.0005$. In addition, the conditional probability score for the partner $(M=$ $.320)$ was higher than that for the best organized of the stimulus people $(M=.178), t(24)=3.56, p<.001$. Finally, the matching index suggested that correct matches were more likely for the partner $(M=2.88)$ than for the stimulus person with the next greatest number of correct matches $(M=2.04), t(24)=2.12$, $p<.04$. These analyses suggest that the results we have summarized on the basis of the average of the other stimulus people are not biased. Even when the partner is compared with the bestrecalled and best-organized other stimulus person, the partner is responded to differently than are the others. ${ }^{3}$

\section{Discussion}

These findings strongly suggest that anticipated-interaction instructions instigate the type of processing that causes the target of the interaction to be individuated and separated from the other stimulus people in memory. This was reflected in both the amount and organization of subjects' free recall, even though they did not anticipate a recall test.

Although the present study suggests that anticipated-interaction instructions can influence the processing of social information, it did not allow us to identify or specify what is special or unique about anticipated-interaction instructions. How do subjects process information about the partner that leads it to be more memorable and better organized than the information about the other stimulus people? Are anticipated-interaction instructions functionally different from impression formation instructions? Srull and Brand (1983) suggested that anticipating an interaction with another person will lead subjects to try to form a clear impression of this target person, and that it is the effort to integrate all of the individual information items about a person to form a clear impression that produces high levels of recall and organization.

Our goal in the following study was to compare anticipatedinteraction instructions with a variety of other processing goal instructions on memory and organization in an effort to determine what, if anything, is special about expecting future interactions with a target person. Specifically, we compared anticipated-interaction instructions with instructions to (a) memorize information about the target, (b) form an impression of the target, (c) compare the target to the self, and (d) compare the target to a best friend. This list of alternative goals is certainly not exhaustive, but each goal has some precedent in the literature and will likely provide some insight into the processes involved in processing information about the target of a prospective interaction.

Some of the processing mechanisms associated with memory and impression formation instructions have been systematically examined in the person memory literature. It has repeatedly been demonstrated that subjects evidence poorer recall for and less organization of stimulus items following memory instructions than when following impression formation instructions (Hamilton et al., 1980; Lichtenstein \& Srull, 1987; Pryor et al., 1982; Srull, 1981, 1983; Wyer \& Gordon, 1982). The recall and organization advantages following impression formation instructions presumably result from processes involved in subjects' attempts to integrate all the information learned about a target into a coherent impression. Memory instructions typically instigate rote memorization processes (Srull, Lichtenstein, \& Rothbart, 1985). If Srull and Brand (1983) were correct in suggesting that anticipated-interaction instructions cause subjects to attempt to integrate stimulus items into coherent impressions of target people, then recall and organization scores should be similar for anticipated-interaction and impression formation subjects, and both of these groups should be higher than the recall and organization scores for memory instruction subjects.

\footnotetext{
${ }^{3}$ In addition to these analyses, we did a series of analyses for nonrandomness (Conover, 1980), assessing whether the proportion of instances the partner was the best remembered or best organized of the five stimulus people was better than chance. The proportion of instances in which the partner was the best remembered (i.e., recall and correct matches) or best organized (i.e., conditional probability) of the five stimulus people was better than chance in all cases. These data corroborate the results summarized in the text.
} 
There are alternatives to the notion that anticipated-interaction instructions function like impression formation instructions. One such alternative is the possibility that in the anticipated-interaction condition, subjects might attempt to compare the information about the target with themselves. Conceivably, subjects may do this in order to search for common points of interest to help facilitate communication with the target person, or they may do this to find potential points of conflict in the interaction that they might want to avoid. By comparing the information about the other person with themselves, they may also inadvertently make the information about the target person more memorable by virtue of having associated the target-person items with self-relevant pieces of information. Extensive research on the processing of self-information has clearly shown that many types of information are better organized and recalled when thought about in relation to the self than when not (for a review, see Kihlstrom et al., 1987).

This explanation is different from the impression formation hypothesis to the extent that the items of information about the target person are not considered in relation to each other but in relation to the self. This alternative suggests that the target-person items may be less likely to be recalled contiguously in the anticipated-interaction and self-comparison conditions than in the impression formation condition. In addition, recall is expected to be greater in the self-comparison condition than in the memory condition owing to the organizational principles that are inherent in the self-knowledge structures and are assumed to be used in this condition to process information about the target.

The self-comparison hypothesis also suggests that the nameto-item association for the target person will not be as strong in the anticipated-interaction condition as in the impression formation condition (because it is assumed that target-person items will have to be considered in relation to the self, not in relation to the target person's name). Indeed, this hypothesis suggests that subjects in the anticipated-interaction or self-comparison conditions will not perform the matching task any better than will subjects in the memory condition.

Finally, it is possible that the information about the target person may not be directly compared with the self in the anticipated-interaction condition, but rather with a familiar other (e.g., best friend) whom the subject is reminded of while processing the information about the target person. In this alternative, the target-person items are considered in relation to features of a salient familiar other, rather than to the self or to the other items about the target person. This scenario suggests that the recall and matching measures obtained from the anticipated-interaction condition may resemble those found in a significant other-comparison condition (again, both of which are assumed to be better than those obtained in the memory set condition, but worse than those obtained in the impression formation condition; see Kihlstrom et al., 1987). This hypothesis, along with the other alternative explanations of the anticipatedinteraction effect, was tested in Study 2.

\section{Study 2}

\section{Method}

Overview. The procedure for Study 2 is identical to that for Study 1 except that subjects did not participate in a telephone interview. During the experiment, however, subjects in the anticipated-interaction condition were led to believe that the other subjects had participated in a previous interview (a manipulation check suggested that subjects believed this). Groups of 6 women participated in the experimental sessions, and the stimulus materials were the same as those used in Study 1. All subjects read through stimulus information about five people at a rate of $6 \mathrm{~s}$ per item and were asked to recall that information, perform a name-to-item matching task, and complete manipulation checks. The free-recall and matching protocols served as the primary dependent measures.

Subjects and design. The 80 subjects received course credit for their participation. The design of the study was an instruction set (anticipated interaction vs. impression formation vs. memory vs. self-comparison vs. friend-comparison) $\times$ stimulus set (one vs. two) $\times$ partner location (second vs. fourth) between-subjects factorial. We randomly assigned 4 subjects to each of the 20 conditions.

The anticipated-interaction instructions were identical to those in Study 1 . In each of the other instruction conditions, a target was identified as the focus of the instruction. Thus, subjects in the impression condition were asked specifically to form an impression of one of the stimulus people to decide what that person would be like. In the memory condition, subjects were instructed to memorize information about one of the stimulus people. In the self- and friend-comparison conditions, subjects were asked to determine how similar one of the stimulus people was to themselves or to their best friend. In each of these other instruction conditions, subjects were told that information about the four other people would be found in the booklet, but they were given no specific instructions concerning the other four stimulus people.

\section{Results}

Manipulation checks. The manipulation check data suggested that our manipulations were successful. Subjects in each of the instruction set conditions were able to accurately report their instructions. Of the subjects, $96 \%$ were able to correctly identify their objective, and $94 \%$ reported that they followed this objective as they read through the stimulus information (the remaining $6 \%$ were either incorrect or provided no response). All of the subjects in the anticipated-interaction condition $(100 \%)$ reported that they believed that they would participate in a two-person, group problem-solving session. When asked with how many others they would participate, $94 \%$ correctly reported that they would work with one other person (1 subject failed to answer this question).

Memory and organization measures. As in Study 1, we were interested in how much information subjects could recall and how that information was organized in subjects' memories. Our primary goal, however, was to compare performance on these measures following anticipated-interaction instructions with the other processing goals included in this study. We compared recall scores, conditional probability scores, and name-to-item matching scores for the target person with the average scores for the remaining four stimulus people. In reporting the following analyses, we refer to the stimulus person singled out for subjects (e.g., the partner in the anticipated-interaction condition, the person about whom subjects were to form an impression, etc.) as the target person.

We first examined subjects' recall of information about their partner relative to their average level of recall across the other four stimulus people. The data were submitted to an instruction set (anticipated interaction vs. impression formation vs. self- 
Table 2

Mean Target and Average Other Recall, Conditional Probability, and Matching Scores as a Function of Instruction Set in Study 2

\begin{tabular}{lccccc}
\hline & \multicolumn{5}{c}{ Instruction set } \\
\cline { 2 - 6 } & $\begin{array}{c}\text { Anticipated } \\
\text { interaction }\end{array}$ & Impression & Self-comparison & Friend-comparison & Memory \\
\hline $\begin{array}{l}\text { Recall } \\
\text { Target }\end{array}$ & $4.38_{\mathrm{a}}$ & $4.00_{\mathrm{a}}$ & $3.31_{\mathrm{b}}$ & $3.38_{\mathrm{b}}$ & $3.00_{\mathrm{b}}$ \\
$\begin{array}{c}\text { Average other } \\
\text { Conditional }\end{array}$ & $1.80_{\mathrm{b}}$ & $2.33_{\mathrm{b}}$ & $2.53_{\mathrm{b}}$ & $2.22_{\mathrm{b}}$ & $2.23_{\mathrm{b}}$ \\
$\quad \begin{array}{l}\text { probability } \\
\text { Target }\end{array}$ & & & & & \\
Average other & $.844_{\mathrm{a}}$ & $.578_{\mathrm{b}}$ & $.594_{\mathrm{b}}$ & $.546_{\mathrm{b}}$ & $.281_{\mathrm{b}}$ \\
Matches & $.328_{\mathrm{b}}$ & $.438_{\mathrm{b}}$ & $.453_{\mathrm{b}}$ & $.380_{\mathrm{b}}$ & $.297_{\mathrm{b}}$ \\
Target & $3.81_{\mathrm{a}}$ & $3.31_{\mathrm{a}}$ & $2.47_{\mathrm{b}}$ & $3.19_{\mathrm{b}}$ & $1.69_{\mathrm{b}}$ \\
Average other & $2.28_{\mathrm{b}}$ & $2.19_{\mathrm{b}}$ & $2.36_{\mathrm{b}}$ & $2.38_{\mathrm{b}}$ & $2.27_{\mathrm{b}}$ \\
\hline
\end{tabular}

Note. Any two means in a given column that do not have the same subscript are different at $p<.05$.

comparison vs. friend-comparison vs. memory) $\times$ stimulus set (one vs. two) $\times$ target location (second vs. fourth) $\times$ recall type (target vs. average others) mixed-model analysis of variance (ANOVA). Instruction set, stimulus set, and partner location were between-subjects variables, and recall type was a withinsubjects variable. Overall, subjects recalled a greater number of items about the target $(M=3.61)$ than about the others $(M=$ 2.22), $F(1,70)=95.51, p<.00001$. Instruction set did not affect recall levels, $F(4,70)=1.79, p<.14$. However, the Instruction Set $\times$ Recall Type interaction was significant, $F(4$, $70)=5.70, p<.0005$. The means for these recall data are presented in Table 2 . Least significant difference tests $(p<.05)$ suggested that anticipated-interaction and impression formation instructions lead to high levels of recall of the target person compared with the average amount recalled about the other stimulus people. Recall for the target was not better than recall for the others in the memory, self-comparison, or other-comparison instruction conditions.

Subjects' conditional probability scores were considered next. We calculated two conditional probability indexes for each subject, one for the target and one for the average conditional probability across the other four stimulus people. We submitted these data to the same mixed-model ANOVA described for the recall data, except that this time the conditional probability scores served as the repeated measure. Conditional probabilities were higher for the target $(M=.57)$ than for the others $(M=.38), F(1,70)=15.50, p<.0002$. The analysis also revealed an instruction set main effect, $F(4,70)=2.63, p<.04$. A post hoc least significant difference test $(p<.05)$ suggested that overall conditional probabilities in the memory condition $(M=.29)$ were lower than conditional probabilities in the anticipated-interaction ( $M=.59$ ), impression $(M=.51)$, self-comparison $(M=.52)$, and friend-comparison $(M=.46)$ conditions, but that the latter four conditions did not differ from each other.

The two main effects described in the preceding paragraph were qualified by a significant Instruction Set $\times$ Conditional Probability Type interaction, $F(4,70)=3.32, p<.02$. The conditional probability means for this interaction are presented in
Table 2. There are two important things to note about these data. First, the conditional probability index for the target is much higher in the anticipated-interaction condition than in any of the other conditions. Second, post hoc least significant difference analyses $(p<.05)$ suggested that only in the anticipated-interaction condition (but not in the other instruction conditions) is conditional probability for the target significantly higher than the average conditional probability for the remaining four stimulus people. Anticipated-interaction instructions, then, seem to exert their strongest influence on the formation of item-to-item associations. Anticipated-interaction instructions apparently lead subjects to consider the target person's stimulus items in relation to one another in working memory such that associative paths are formed among these items (Srull, 1981).

Finally, we examined the matching data, which provide a measure of the name-to-item associations. The data were submitted to the same mixed-model ANOVA used in the previous analyses (with match type as the repeated measure). We dropped 1 subject from this analysis because she failed to complete the matching task. Overall, correct matches were higher for the target $(M=2.90)$ than for the other stimulus people $(M=2.29), F(1,69)=13.33, p<.0005$. Instruction set did not strongly affect matching levels, $F(4,69)=2.03, p<.10$. Most important, the interaction between instruction set and match type was significant, $F(4,69)=5.22, p<.001$. The means for this interaction are presented in Table 2. Least significant difference analyses $(p<.05)$ tested whether the correct matches for the target were significantly different than the average correct matches for the remaining four stimulus people within each of the five instruction sets. Significant differences arose only in the anticipated-interaction and impression formation conditions. Subjects in the anticipated-interaction and impression formation conditions were more accurate in matching the target's name to her stimulus items than they were in matching the other stimulus people's names to their stimulus items. Subjects' matching performance in the memory, self-comparison, and friend-comparison conditions were equally good for the target and for the other stimulus persons.

As in Study 1, we performed a series of additional analyses 
on just the anticipated-interaction subjects. Specifically, we compared the partner with the best remembered and the best organized of the other stimulus people in order to ensure that we obtained the least biased comparison possible. These analyses indicated that the results we have summarized can be directly interpreted. Recall for the partner was higher $(M=4.38)$ than for the best remembered of the other stimulus persons $(M=$ $1.94), t(16)=7.14, p<.0001$. The conditional probability score for the partner $(M=.844)$ was higher than that for the bestorganized other stimulus person $(M=.469), t(16)=2.16, p<$ .04 . Finally, the matching index suggested that correct matches were more likely for the partner $(M=3.81)$ than for the stimulus person with the next greatest number of correct matches $(M=2.44), t(16)=3.02, p<.008$.

We also performed these same analyses for impression formation subjects. Replicating the impression condition results, impression subjects recalled a greater number of items about the target $(M=4.00)$ than about the best-recalled other stimulus person $(M=2.81), t(16)=4.90, p<.0002$. The conditional probability score for the target $(M=.578)$, however, was not higher than the conditional probability score for the best-organized other stimulus person $(M=.609), t(16)=-0.25, p<.80$. Finally, impression subjects' matching scores were higher for the target $(M=3.31)$ than for the stimulus person with the next greatest number of matches $(M=2.44), t(16)=2.00, p<.06 .^{4}$

\section{General Discussion}

\section{Summary of Findings}

Study 1 demonstrated that subjects, when anticipating an interaction with one of many stimulus people, are more likely to remember more information about the target relative to the remaining stimulus people, to individuate the target in memory, and to form more name-to-item associations for the target than for the other stimulus people. In Study 2, we compared the effect of anticipated-interaction instructions on subjects' recall and organization of information about a target person versus several other stimulus people with a variety of other instructional goals. Considering only the matching and recall data, it appears that anticipated-interaction instructions produce memory effects similar to those produced by instructions to form an impression of the target person. Both of these instructions produced higher levels of recall and of correct matches for the target than for the average stimulus person, something that was not true with the self-comparison, friend-comparison, or memory instructions. This finding is in line with Srull and Brand's (1983) suggestion that when subjects expect to interact with a target person they will try to form a clear impression of that person.

The conditional probability data, however, suggest that anticipated-interaction instructions are doing something more than are impression formation instructions. That is, although recall and matching levels are similar in both conditions, anticipatedinteraction instructions appear to lead to greater individuation of the target from the other stimulus persons. Not only is information about the target well-recalled compared with that about the other stimulus people, but it is also more organized than the information about the others. Unlike previous research that has addressed the issue of whether subjects can individuate targets (e.g., Srull \& Brand, 1983), our research demonstrates that subjects can selectively individuate a target within a group.

Is there anything unique about anticipated-interaction instructions? The present data suggest an affirmative response to this question. In previous theorizing, forming person impressions was expected to lead to high levels of recall and well-organized representations (Hamilton et al., 1980; Hastie, 1980; Srull, 1981; Srull et al., 1985). One goal of the present research was to examine several different indexes that would reflect the nature of the underlying memory representations. Our data suggest that the matching and conditional probability indexes tap different aspects of the structure of memory representations about people (Pryor \& Ostrom, 1981). That is, the matching data appear to be sensitive to the formation of name-to-item associations. The present data suggest that both anticipated-interaction and impression formation instructions are effective in establishing this type of association. The conditional probability index, however, is sensitive not only to the formation of name-to-item associations, but also to the formation of itemto-item associations. The data from Study 2 suggested that anticipated-interaction instructions increase the likelihood that subjects will form not only name-to-item associations, but also item-to-item associations reflected in the high conditional probability scores for the target. Thus, it appears that subjects given instructions to anticipate interacting with a target attempt to compare items learned about the target person with one another, probably in working memory, and thus integrate the information into a well-organized cognitive structure.

These data suggest that it is possible that anticipated-interaction instructions involve more than simply forming an impression of another. They produce a memory structure in which items are associated with a general person node and also with one another. Such a memory structure could have implications beyond the likelihood of producing high levels of recall and good name-to-item matching performance. For example, if subjects were asked to make subsequent judgments about a target person with whom they expected to interact, the well-integrated nature of the memory structure may have led them to be more efficient (faster) in making such judgments as compared with subjects who have a less well-organized memory structure (e.g., impression formation or memory instruction subjects). Recall follows a random and sequential search process (fastie, 1980; Srull et al., 1985). That is, subjects should be able to more quickly access judgment-relevant information in the well-integrated memory structure as spreading activation can proceed

\footnotetext{
${ }^{4}$ As in Study 1, in addition to these analyses we did a series of analyses for nonrandomness assessing whether the proportion of instances that the partner was the best remembered or best organized of the five stimulus people was better than chance. Replicating Study 1, with regard to the anticipated-interaction condition we found that the proportion of instances in which the partner was the best remembered (i.e., recall and correct matches) or best organized (i.e., conditional probability) of the five stimulus people was better than chance in all cases. For the impression formation condition, however, the target was the best recalled (i.e., recall and correct matches) but not the best organized (i.e., conditional probability). These data completely corroborate the findings presented in the text.
} 
from item to item within the network without having to return to the general person node (Wyer \& Carlston, 1979). 'However, accessing judgment-relevant information should take longer when subjects must return to the general person node at retrieval, leading to longer judgment latencies, as would be the case with less well-organized memory structures.

Is the difference between anticipated-interaction and impression formation instructions qualitative or quantitative? At present, it may be important to consider the possibility that, rather than producing qualitatively different structures, anticipatedinteraction and impression formation instructions lead to only quantitative differences in the strength of item-to-item associations. That is, if our impression formation instructions had more strongly stressed the importance of the accuracy of the impression, perhaps subjects would have attempted more itemto-item comparisons in working memory and developed a more integrated memory representation. With the present stimulus materials, we believe that this would not have occurred. Subjects could form accurate impressions without comparing stimulus items with each other.

In forming a global impression of a stimulus person, one may need only to evaluate the overall favorableness or unfavorableness of each stimulus item for the developing impression. That is, each item can be compared with the judgment criterion, and the judgment can be updated without comparing each new item to already learned items. As a result, each item may be associated with the general person node, but not with other stimulus items. In previous research in which the subjects' goal was to form an impression of a stimulus person, the only circumstances under which item-to-item associations were formed was when subjects were presented with inconsistent stimulus information that somehow had to be resolved (Hastie \& Kumar, 1979; Srull, 1981; Srull et al., 1985). Under these circumstances, inconsistent items were compared in working memory with previously learned consistent (as well as subsequently learned consistent and inconsistent) items, which led to the formation of item-to-item associations. In these person memory models, it is unlikely that subjects form item-to-item associations between consistent stimulus items. Such items simply provide confirmation for the developing impression (i.e., they are not uniquely informative about the stimulus person and thus do not require any special elaboration or explanation).

In summary, when the instruction is to form a clear impression of a person, well-integrated memory representations (e.g., many item-to-item associations) seem to follow only when one has some unexpected or unusual information to explain. Otherwise, items seem to be associated with a general person node, but not with each other (Hastie, 1980; Srull et al., 1985). This type of representation would, of course, lead us to expect good performance on a name-to-item matching task, but not high conditional probability scores.

We would not argue, however, that the only condition under which people form integrated representations is when there is some inconsistent or unusual item that needs to be explained. For example, Hastie (1984) suggested that manipulations that increase subjects' outcome dependency on another person encourage subjects to engage in more elaborated processing of information about a stimulus person. Anticipated-interaction instructions, which make subjects outcome-dependent on their partner, may elicit more individuating processes (i.e., formation of item-to-item associations) than impression formation instructions (see also Neuberg \& Fiske, 1987). The present findings suggest that this' is a possibility. Erber and Fiske (1984) ckamined the joint impact of outcome dependency and inconsistency and found that outcome dependency increased subjects' attention only to inconsistent information. Their findings, however, do not preclude the possibility that integrated representations could be formed when there is nothing particularly "consistent" or "inconsistent" in the stimulus materials.

Although we have argued that anticipated-interaction instructions produce memory representations that are qualitatively different than impression formation instructions, the alternative that anticipated-interaction instructions reflect impression formation instructions plus a high level of motivation is not fully ruled out by our data. A study designed to compare the present anticipated-interaction and impression formation conditions with impression formation instructions coupled with varying degrees of motivation would satisfactorily address this possibility. ${ }^{5}$

Name-to-item and item-to-item associations as determinants of recall. What role do name-to-item and item-to-item associations play in determining overall recall levels? Total recall scores were equally high in the impression formation and anticipatedinteraction conditions. The present data suggest that both matching (i.e., name-to-item associations) and organization (i.e., item-to-item associations) can produce high levels of recall. Although speculative, we would argue that neither nameto-item associations nor item-to-item associations are necessary for good recall performance, but that either type of association is sufficient to produce high recall. What would be needed to support this speculation, however, are recall data in which subjects manifest poor name-to-item associations but good item-to-item associations, or vice versa. This type of recall pattern considered in the context of our impression formation condition (high name-to-item associations) and anticipated-interaction condition (high name-to-item and high item-to-item associations) would provide evidence to support the sufficiency argument we summarized earlier.

In the present study, it is difficult to detect the exact role of name-to-item and item-to-item associations in overall recall levels. It seems possible that they could have an additive effect on recall such that when both are present the highest levels of recall would be detected. Recall levels for the target were extremely high (at least $80 \%$ ) in the impression formation and anticipated-interaction conditions of Study 2 . However, subjects were presented with only five items of information about

\footnotetext{
${ }^{5}$ To further explore the possible differences between anticipated-interaction and impression formation instructions, we conducted a series of single degree of freedom interaction tests on the recall, conditional probability, and matching data. The theoretically most meaningful comparison involved the test on the conditional probability data. This interaction was significant, $F(1,28)=9.49, p<.0005$. The interaction revealed that highest levels of organization were evident for the target in the anticipated-interaction condition (see Table 2). There was no significant interaction on the matching data, $F(1,28)=0.70, p<.41$. Finally, the recall data interaction was significant, $F(1,28)=5.58, p<$ .03 , although of no particular theoretical interest.
} 
each person, and the high recall levels for the target could reflect a ceiling effect in the impression formation and anticipated-interaction conditions. If name-to-item associations and item-toitem associations have additive effects on recall, subjects would have to be presented with many more than five items about the stimulus people for such an effect to be detected.

\section{Anticipated Interaction: Scratching the Surface}

We fully expect that the anticipated-interaction construct is more complex than is represented in the present research. We believe that our research has only scratched the surface of some very important ways in which anticipated interaction affects social information processing. That is, the ultimate effect of anticipated-interaction instructions may depend in important ways on who is the target of the interaction (e.g., unknown other, member of liked vs. disliked group), the time frame of the anticipated interaction (e.g., short-term vs. long-term outcome dependency), and the likely consequences of the interaction (e.g., obtaining rewards, avoiding punishments, maintaining status quo). We have argued that subjects, who are involved in a shortterm outcome dependency situation and know very little about their prospective partners, will attempt to form an integrated representation of their partner. As an extreme but very compelling counterexample, consider the following situation. ${ }^{6}$

Imagine a Ku Klux Klan member who anticipates interacting with a Black man. Will the Klan member individuate this target in memory? Although it is possible, it may not be likely. It may be more likely that anticipated interaction in this setting will make salient to the perceiver a need to preserve the strongly held stereotypes and prejudices against Blacks. Consequently, rather than noticing the features that make this person unique, the perceiver may attend to and remember a disproportionate amount of stereotype-consistent information. Inconsistent information may be ignored or rationalized away. Moreover, the perceiver may incorrectly "remember" stereotype-consistent information that was never encountered. This type of information processing following anticipated interaction is likely to be category-based rather than person-based, as was the case in the present study.

In summary, we would suggest that anticipated-interaction instructions can potentially instigate a variety of different goals depending on both situational and personality factors. These different types of goals that can follow from anticipating an interaction with a person will likely have direct implications for the nature of the underlying memory representation of the target of the interaction. We do not believe that the complexity of anticipated-interaction instructions undermines the importance of the present findings. Our findings suggest that anticipated-interaction instructions can contribute something unique to person memory representations. That anticipatedinteraction instructions could work in different ways in different settings is an exciting possibility that should lead to productive new research directions.

\section{Comparison Processes: Less Efficient Organization Instructions}

Interestingly, the self-comparison and best-friend comparison do not appear to lead to any advantage of the target of the comparison over the other stimulus people in terms of number of items recalled, number of correct matches, or conditional probabilities. This is somewhat surprising, especially in the selfcomparison condition, given that it has been repeatedly demonstrated that encoding information with respect to the self leads to better memory performance for that information than for information that is otherwise encoded (see Kihlstrom et al., 1987). There are, however, differences between the present research and the standard self-reference effect research. In the self-reference research, subjects are typically asked to make yes-no judgments about the self-descriptiveness of trait adjectives. This type of task is much more a self-focus task than is the present task, in which the primary goal is to make judgments about another person. It appears that the type of comparison processes involved in the present task do not produce the same type of processing that enables the self to function as an effective retrieval device. This type of analysis holds for the friend-comparison condition as well.

In addition, Klein and Kihlstrom (1986) have argued that organization rather than self-reference per se accounts for the established high levels of recall for information encoded in a self-referential fashion. They demonstrated in a series of experiments that self-referent and semantic encodings produce equal recall levels when they are first equated for the amount of organization they encourage. Even though self-referent encoding can produce high levels of recall through organization of the stimulus information in memory, anticipated-interaction instructions induce subjects to develop a more strongly organized structure than that produced by the self-comparison process of Study 2.

\section{Conclusion}

The present set of studies highlights the importance of exploring the role of goals in social information processing. These studies follow in the growing tradition of efforts to integrate motivational and cognitive perspectives on social perception processes. The goals that individuals bring to bear on the processing of information about others have implications for how much information is remembered about the others and how that information is organized in memory. These cognitive consequences of goal-directed processing about people will likely affect future judgments about and interactions with the individuals. We have examined only a few of the potential set of goals that affects perceivers' social information processing. In fact, we believe that developing a taxonomy of goals that perceivers bring to bear on social information processing and then systematically investigating how such goals affect memory structures and processes should be a priority for research in social cognition.

\footnotetext{
${ }^{6}$ We thank an anonymous reviewer for suggesting this example.
}

\section{References}

Berscheid, E., Boye, D., \& Darley, J. M. (1968). The effect of forced association upon voluntary choice to associate. Journal of Personality and Social Psychology, 8, 13-18.

Berscheid, E., Graziano, W., Monson, T., \& Dermer, M. (1976). Out- 
come dependency, attention, attribution, and attraction. Journal of Personality and Social Psychology, 34, 987-989.

Conover, W. J. (1980). Practical nonparametric statistics (2nd ed.). New York: Wiley.

Erber, R., \& Fiske, S. T. (1984). Outcome dependency and attention to inconsistent information. Journal of Personality and Social Psychology, 47, 709-726.

Hamilton, D. L., Katz, L. B., \& Leirer, V. O. (1980). Organizational processes in impression formation. In R. Hastie, T. M. Ostrom, E. B. Ebbesen, R. S. Wyer, D. L. Hamilton, \& D. E. Carlston (Eds.), Person memory: The cognitive basis of social perception (pp. 121-154). Hillsdale, NJ: Erlbaum.

Harvey, J. H., Yarkin, K. L., Lightner, J. M., \& Town, J. P. (1980). Unsolicited interpretation and recall of interpersonal events. Journal of Personality and Social Psychology, 38, 551-568.

Hastie, R. (1980). Memory for behavioral information that confirms or contradicts a personality impression. In R. Hastie, T. M. Ostrom, E. B. Ebbeson, R. S. Wyer, D. L. Hamilton, \& D. E. Carlston (Eds.), Person memory: The cognitive basis of social perception (pp. 155177). Hillsdale, NJ: Erlbaum.

Hastie, R. (1984). Causes and effects of causal attribution. Journal of Personality and Social Psychology, 46, 44-56.

Hastie, R., \& Kumar, A. P. (1979). Person memory: Personality traits as organizing principles in memory for behaviors. Journal of Personality and Social Psychology, 37, 25-38.

Hastie, R., Park, B., \& Weber, R. (1984). Social memory. In R. S. Wyer \& T. K. Srull (Eds.), Handbook of social cognition (Vol. I, pp. 151212). Hillsdale, NJ: Erlbaum.

Kihlstrom, J. F., Cantor, N., Albright, J. S., Chew, B. R., Klein, S. B., \& Neidenthal, P. M. (1987). Information processing and the study of the self. In L. Berkowitz (Ed.), Advances in experimental social psychology (Vol. 21, pp. 145-177). New York: Academic Press.

Klein, S. B., \& Kihlstrom, J. F. (1986). Elaboration, organization, and the self-reference effect in memory. Journal of Experimental Psychology: General, 115, 26-38.

Layton, R. E., \& Insko, S. (1974). Anticipated interaction and the similarity-attraction effect. Sociometry, 37, 149-162.

Lichtenstein, M., \& Srull, T. K. (1987). Processing objectives as a determinant of the relationship between recall and judgment. Journal of Experimental Social Psychology, 23, 93-118.

Neuberg, S. L., \& Fiske, S. T. (1987). Motivational influences on impres- sion formation: Outcome dependency, accuracy-driven attention, and individuation processes. Journal of Personality and Social Psychology, 53, 431-444.

Pryor, J. B., \& Ostrom, T. M. (1981). The cognitive organization of social information: A converging operations approach. Journal of Personality and Social Psychology, 41, 628-641.

Pryor, J. B., Simpson, D. D., Mitchell, M., Ostrom, T. M., \& Lydon, J. (1982). Structural selectivity in the retrieval of social information. Social Cognition, 1, 336-357.

Sorrentino, R. M., \& Higgins, E. T. (1986). Handbook of motivation and cognition: Foundations of social behavior. New York: Guilford Press.

Srull, T. K. (1981). Person memory: Some tests of associative storage and retrieval models. Journal of Experimental Psychology: Human Learning and Memory, 7, 440-463.

Srull, T. K. (1983). Organizational and retrieval processes in person memory: An examination of processing objectives, presentation format, and the possible role of self-generated retrieval cues. Journal of Personality and Social Psychology, 44, 1157-1170.

Srull, T. K., \& Brand, J. F. (1983). Memory for information about persons: The effect of encoding operations upon subsequent retrieval. Journal of Verbal Learning and Verbal Behavior, 22, 219-230.

Srull, T. K., Lichtenstein, M., \& Rothbart, M. (1985). Associative storage and retrieval processes in person memory. Journal of Experimental Psychology: Learning, Memory, and Cognition, 11, 316-345.

Srull, T. K., \& Wyer, R. S. (1986). The role of chronic and temporary goals in social information processing. In R. M. Sorrentino \& E. T. Higgins (Eds.), Handbook of motivation and cognition: Foundations of social behavior (pp. 503-549). New York: Guilford Press.

Tyler, T. R., \& Sears, D. O. (1977). Coming to like obnoxious people when we must live with them. Journal of Personality and Social Psychology, 35, 200-211.

Wyer, R. S., \& Carlston, D. E. (1979). Social cognition, inference, and attribution. Hillsdale, NJ: Erlbaum.

Wyer, R. S., \& Gordon, S. (1982). The recall of information about persons and groups. Journal of Experimental Social Psychology, 18, 128164.

Received November 30, 1987

Revision received October 3, 1988

Accepted October 6, 1988 